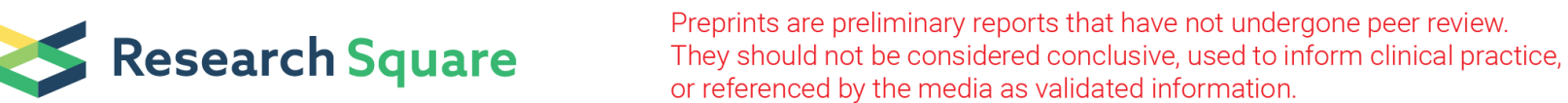

\section{Role of NRF2 cascade in determining the differential response of cervical cancer cells to anticancer drugs- an in vitro study}

Pushkal Sinduvadi Ramesh

JSS Academy of Higher Education and Research

Sharanya Raja

JSS Academy of Higher Education and Research

Shwethambari Harave Udayakumar

JSS Academy of Higher Education and Research

Shruthi Chandrashekar

JSS Academy of Higher Education and Research

Suma M Nataraj

JSS Academy of Higher Education and Research

Devananda Devegowda ( $\nabla$ devanandd@jssuni.edu.in )

JSS Academy of Higher Education and Research https://orcid.org/0000-0003-0577-4622

\section{Research Article}

Keywords: NRF2, cervical cancer, chemoresistance, antioxidant genes, reactive oxygen species, gene expression

Posted Date: September 27th, 2021

DOI: https://doi.org/10.21203/rs.3.rs-844951/v2

License: (9) This work is licensed under a Creative Commons Attribution 4.0 International License.

Read Full License

Version of Record: A version of this preprint was published at Molecular Biology Reports on October 21st, 2021. See the published version at https://doi.org/10.1007/s11033-021-06848-2. 


\section{Abstract \\ Background}

Cervical cancers are usually treatable if detected in early stages by a combination of therapies. However, the prognosis of cervical cancer patients with metastasis remains unfavorable due to the fact that most of the cervical carcinomas are either resistant to anticancer drugs or show signs of relapse after initial treatment. Therefore, it is important to control the chemoresistance as it is the key to develop effective treatment options for cervical cancer.

\section{Objective}

The current study aimed at evaluating the differential responses of cervical cancer cells to anti-cancer drugs and assessed whether the differences in the expression profiles of antioxidant genes regulated by NRF2 (nuclear factor erythroid-2-related factor 2), led to the variations in the sensitivities of the cancer cells to treatment. Methodology. Three cervical cancer cell lines were investigated for their differences in NRF2 pathway by measuring the gene expression and enzyme activity. The differences in the sensitivity to anti-cancer drugs and variation in ROS profile was also evaluated. The addition of exogenous drugs to manipulate the intracellular ROS and its effect on NRF2 pathway genes was also investigated.

\section{Results}

HeLa and SiHa cells were more sensitive to cisplatin and oxaliplatin treatment than C33A cells. HeLa and SiHa cells had significantly lower NRF2 gene levels, NQ01 enzyme activity and basal GSH levels than C33A cells. Levels of ROS induced were higher in HeLa than C33A cells.

\section{Conclusion}

Overall, the differences in the cellular levels of antioxidant regulatory genes led to the differential response of cervical cancer cells to anti-cancer drugs.

\section{Introduction}

Cervical cancer is the third most common malignancy among women; with nearly 570,000 women being affected every year worldwide, of which approximately half the affected population die [1, 2]. Research till date has pointed that over $90 \%$ of the cervical cancer cases are caused by the high risk Human Papillomavirus infection leading to carcinogenesis [2,3]. Cervical carcinomas are usually treatable if detected in early stages or pre-invasive phases by a combination of chemotherapy, radiotherapy and surgery. However, the prognosis of cervical cancer patients with metastatic disease remains unfavorable due to the fact that most of the cervical carcinomas are either resistant to anticancer drugs or show signs 
of relapse after initial treatment [4-6]. Therefore, the control of chemoresistance is the key to develop effective treatment options for cervical cancer and exploring new markers and pathways for therapeutic interventions are of utmost importance.

There are many factors that are responsible for chemoresistance including the individual's genetic makeup, epigenetics, and lifestyle factors. Another reason for chemoresistance in cancer cells is mainly due to the activation of survival pathways that aid cells to escape from treatment induced cell death [710]. One such significant cytoprotective pathway is regulated by a transcription factor NRF2. NRF2 (nuclear factor erythroid-2-related factor 2) or NFE2L2 (nuclear factor erythroid-derived 2-like 2) is a basic leucine zipper protein and a transcription factor that regulates various cytoprotective genes aiding the cells to counteract the oxidative and electrophilic stress [11].

Under homeostatic conditions, NRF2 is bound to its antagonist protein KEAP1 (Kelch-like ECH-associated protein 1) in the cytoplasm, which directs it to ubiquitin mediated proteasomal degradation (Fig. 1). When the cells encounter ROS or electrophiles, KEAP1 undergoes conformational change resulting in the release and stabilization of NRF2. This stabilized NRF2 then translocates to the nucleus, where it binds to ARE (antioxidant response element) region on the promoter and transcribes its downstream genes coding for antioxidant proteins and detoxifying enzymes [11-13]. Research has provided enough evidence to demonstrate that activation of NRF2 provides robust protection from oxidative damage to the cells by directly or indirectly detoxifying ROS $[14,15]$. Therefore, cells show increased sensitivity when NRF2 is knocked down and in contrast cells exhibit resistance to cell death when KEAP1 is knocked down [16].

Mechanistic studies have shown that cancer cells in order to tackle the ROS generated due to metabolic stress, acquires gain of NRF2 function. Singh et al. in a series of studies demonstrated that there is a gain-of-function mutation in NRF2 pathway in NSCLC (non-small cell lung cancer) tumor tissues. The same group also showed that either pharmacological inhibition of NRF2 cascade or knockdown of NRF2 by RNAi in cancer cells sensitizes them to ROS-induced cell death upon exposure to chemotherapeutic drugs $[17,18]$. Further, they also reported that the poor survival of NSCLC patients correlates with hyperactivation NRF2. Zhang et al. showed that high NRF2 expression in hepatocellular carcinoma was associated with poor prognosis [19]. Concordantly, Tong et al. demonstrated that low expression of NRF2 and NQ01 protein by immunohistochemistry in tumors was a predictor of better survival in patients with NSCLC [20].

Recent studies have shown that the upregulation of HMOX-1 (Heme oxygenase 1) and NQO1 (NADPH dehydrogenase quinone 1) confer tumor cell survival and inhibits apoptosis by modulating cellular GSH levels [21, 22]. NQO1 helps to detoxify the cancer drugs, whereas HMOX-1 contributes to cyto-protection against the drug induced oxidative stress. These findings suggest that activation of NRF2 is linked to chemoresistance. Thus the upregulation of cytoprotective genes may increase the cell proliferation by increasing the chemoresistance or by rapidly removing the anticancer drugs from the system. It has also been shown that depleting GSH levels have sensitized cancer cells to various chemotherapeutic agents. This shows NRF2 and its target genes favor the cancer cells to tackle the ROS produced by the drugs, 
providing resistance. Therefore studying the NRF2 cascade and understanding them in order to use them as targets can prove to be beneficial to overcome the resistance. The current study aimed at evaluating the differential responses of cervical cancer cells to anti-cancer drugs and assessed whether the differences in the expression profiles of antioxidant genes regulated by NRF2 as well as contrasting ROS profiles led to the variations in the sensitivities of the cancer cells to treatment.

\section{Results}

\section{HeLa and SiHa were more sensitive to cisplatin and oxaliplatin treatment than C33A}

Although the potent inhibitory activity of anti-cancer agents on cervical cancer cells is well known, we wanted to assess the differential response to treatment in various cells derived from the carcinoma of cervix. To evaluate and compare the sensitivity of HeLa, $\mathrm{SiHa}$ and C33A a dose dependent cytotoxicity assay was performed with Cisplatin and Oxaliplatin. With both the treatments the data demonstrated a dose dependent cell growth inhibition with $\mathrm{HeLa}$ and $\mathrm{SiHa}$ cells exhibiting more sensitivity to treatment when compared to C33A cells 48 hours post treatment (Figure 2A, 2B and 2C). It was also found that C33A and HeLa cells were more resistant to Oxaliplatin when compared to treatment with Cisplatin. On the other hand, SiHa was found to be more resistant to cisplatin and showed dose dependent cell growth inhibition with oxaliplatin. Overall C33A was quiet resistant to both cisplatin and oxaliplatin when compared to HeLa and SiHa which showed more growth inhibition to anti-cancer drug treatment.

\section{HeLa and SiHa cells have significantly lower NRF2 levels than C33A cells}

NRF2 is a transcription factor which regulates antioxidant genes and thereby affects the sensitivity of the cancer cells to therapeutic agents. Since the cell lines in our study showed differential response to the drugs, we evaluated the NRF2 pathway by measuring the gene levels of NRF2 and its target genes using quantitative real time PCR. This experiment was performed to understand whether the differences in NRF2 pathway made the cells either resistant or sensitive to the chemotherapeutic treatment.

The data demonstrated that the expression of NRF2 and NQ01 genes were significantly lower in SiHa and HeLa cells when compared to C33A cells (Figure 3A). KEAP-1 gene which is considered to be a negative regulator of NRF2 was significantly higher in HeLa and SiHa cells in contrast to C33A cells (Figure 3B). HMOX-1 gene even though is a downstream gene of transcription factor NRF2, did not show any trend in differences between the cells probably as it is involved in plethora of subordinate pathways (Figure 3C). However, GCLC and GCLM gene expressions were relatively higher in SiHa cells followed by C33A and lowest in HeLa cells (Figure 3D).

\section{HeLa and SiHa cells have significantly lower NQ01 activity and basal GSH levels compared to C33A cells}

A standard read-out parameter to assess NRF2 pathway is to measure NQ01 enzyme activity. We performed NQO1 activity in the cell lysates of $\mathrm{SiHa}$, HeLa cells and C33A cells. The trend followed in the gene expression of NQ01 was also observed in the enzyme activity assay with C33A cells showing 
highest NQO1 activity of $44.87 \pm 6.22 \mu \mathrm{mol} / \mathrm{min} / \mathrm{mg}$ protein and SiHa and HeLa cells showed lower NQ01 activity of $7.02 \pm 1.28 \mu \mathrm{mol} / \mathrm{min} / \mathrm{mg}$ protein and $3.4 \pm 0.65 \mu \mathrm{mol} / \mathrm{min} / \mathrm{mg}$ protein respectively (Figure $4 \mathrm{~A}$ ).

Our gene expression analysis revealed that GCLC and GCLM genes that are important to catalyze the rate limiting step of GSH production is relatively higher in SiHa followed by C33A and HeLa. Therefore we measured total GSH levels in these cells and found that the cells having low NRF2/NQO1 activity also had low GSH levels corroborating with the existing evidence emphasizing on high oxidative stress levels in those cells. C33A cells showed GSH levels of $15.2 \pm 4.81 \mu \mathrm{M}$ whereas $\mathrm{SiHa}$ and HeLa cells showed GSH levels of $5.11 \pm 1.65 \mu \mathrm{M}$ and $7.11 \pm 1.53 \mu \mathrm{M}$ respectively (Figure 4B).

\section{Levels of ROS induced is higher in HeLa than C33A cells}

The differential expression of antioxidant related genes regulated by NRF2 is most likely to influence the baseline levels of Reactive Oxygen Species (ROS) in these cells. To test this idea, C33A and Hela were stained with H2DCFDA (2', 7- dicholofluorescin diacetate) after treating with selected drugs along with a positive control and the excitation-emission spectrum was measured using a multimode plate reader.

$\mathrm{H}_{2} \mathrm{O}_{2}$ induced significantly higher ROS levels in both HeLa and C33A cell lines when compared to respective controls. Cisplatin and Oxaliplatin did induce ROS levels higher than the control cells but not significant enough with respect to the positive control in C33A cells (Figure 5A). In contrast, HeLa cells treated with $100 \mu \mathrm{M}$ of Cisplatin showed significant induction of a higher ROS with respect to control as well as the positive control. However, Oxaliplatin failed to induce any significant amount ROS when compared to control and positive control (Figure 5B). These data suggested that cells with an elevated level of ROS are more susceptible to treatment and concurring to it our experiments showed HeLa cells with higher ROS levels was more sensitive to anti-cancer drugs when compared to C33A cells.

\section{Treatment with N-Acetyl Cysteine (NAC) but not L-Buthionine Sulfoximine (L-BSO) elevates NRF2 and its downstream genes}

To assess whether the internal levels of oxidative stress affects the cellular sensitivity to chemotherapeutic agents, we treated HeLa cells with L-Buthionine Sulfoximine (L-BSO), a known glutathione inhibitor and N-Acetyl Cysteine (NAC) a known exogenous antioxidant. A dose of $20 \mu \mathrm{M}$ of LBSO and $4 \mathrm{mM}$ of NAC was used for treating HeLa cells in a 6 well plate as per literature. After 12 hours of treatment, cells were collected for extraction of RNA and subjected to gene expression analysis. The data demonstrated that NAC treatment significantly elevated NRF2 and its downstream genes NQ01, GCLM, GCLC and HMOX-1. Treatment with BSO elevated only NRF2 gene level slightly higher than control, but the rest of the genes showed no evidence of significant increase. On the other hand, KEAP-1 did not have any significant effect on its gene expression with either of the treatments (Figure 6). Together these 
experiments demonstrated that modifying the internal levels of oxidative stress can bring about the change in the antioxidant regulating genes and thereby can dictate the terms of cellular response to anticancer agents.

\section{Discussion}

Resistance to chemotherapeutic drugs or their differential response is a major concern in the treatment of cervical cancer and other cancers in general. It has been shown by various studies that this resistance is due to alterations in the activation of survival pathways that enables cancer cells to escape from treatment induce cell death. One such cell survival pathway is regulated by the transcription factor NRF2. Elucidation of such pathways and studying them will help us to identify new treatment options and explore new biomarkers.

In this study, we asked whether there is any differential responses of cervical cancer cells to FDA approved anti-cancer drugs such as Cisplatin and Oxaliplatin. We selected C33A, SiHa and HeLa as representatives of cervical carcinoma. In the first phase of experiment, all three cell lines were subjected to cell viability assay after treating with anti-cancer drugs for 48 hours. The treatment sensitivities were compared amongst the three cell lines and overall it was found that C33A was quiet resistant to both cisplatin and oxaliplatin when compared to HeLa and SiHa which showed more growth inhibition to treatment. Also, C33A and HeLa cells were more resistant to Oxaliplatin when compared to SiHa, whereas, SiHa was found to be more resistant to Cisplatin. A similar study compared and evaluated the sensitivity of SiHa and CaSki cells to Cisplatin and Doxorubicin [23]. The study demonstrated that CaSki was relatively sensitive to both the treatments when compared with SiHa cells which showed resistance to the anti-cancer drugs which was in concordance with our study.

Gene expression of NRF2 signaling pathway was assessed to check if NRF2 and its downstream genes were responsible for the differential response. We found out that C33A cells had high levels of NRF2 and NQO1 when compared to SiHa and HeLa. In concordance with the cytotoxicity profile, we found that $\mathrm{SiHa}$ and HeLa with relatively less NRF2 and its target gene levels to be more sensitive to anti-cancer agents than C33A with higher gene levels. In contrast to our results, a study assessing the unique secretome compositions contributing to the tumor development reported relatively higher levels of NRF2 in both $\mathrm{SiHa}$ and C33A [24]. Recent studies have demonstrated that oxidative stress pathways and antioxidant defense contribute to drug resistance in cancer. A study by Cho, Jeong-Min, et al demonstrated that when compared to wild type cells the NRF2 deficient cells were more sensitive to cisplatin mediated cell death [25]. The reverse of it was proved true in a study where it was demonstrated that NRF2 knockdown using shRNA inhibited tumor growth and increased the efficacy of chemotherapy in cervical cancer cells [26]. In $\mathrm{SiHa}$ and HeLa cell lines the expression of KEAP1 was high and a higher level of KEAP1 is known to reduce the NRF2 levels [27]. In concordance with the gene levels, NQO1 enzyme activity (a surrogate marker of NRF2 pathway) and GSH levels also showed higher levels in C33A when compared to SiHa and HeLa. Higher levels of NQ01 and GSH means higher the resistance to anti-cancer drugs. Plethora of studies has already provided evidence citing the molecular mechanisms behind the resistance developed 
by cancers to platinum drugs and one among them is $\mathrm{GSH}$, a thiol containing tripeptide which can bind to cisplatin and prevent the later from binding to DNA and hence reduce cell death [28].

Further we analyzed the basal levels of intracellular ROS and its levels after treating with Cisplatin and Oxaliplatin. It was quite evident that the differential expression of antioxidant related genes regulated by NRF2 influenced the baseline levels of ROS in these cells. Results showed that the HeLa cells had relatively higher ROS levels when compared to C33A cells which made the former more sensitive to anticancer drugs. This indicates that high levels of NRF2 in C33A could have aided in reducing the drug induced ROS levels and hence made them resistant to the anti-cancer drug treatment. Studies have showed the protective capacity of NRF2 can be hijacked by the cancer cells which will in turn provide the cancer cells with increased cell growth and drug resistance $[29,30]$. These results are consistent with a similar study which shows that the treatment with Doxorubicin and Cisplatin led to increase in ROS levels and produced different profiles of ROS [23].

To examine whether the internal levels of oxidative stress affects the antioxidant genes regulating the cellular sensitivity to chemotherapeutic agents we depleted ROS levels by adding a known antioxidant i.e., NAC and also increasing ROS levels by adding a known glutathione inhibitor L-BSO in HeLa cells. The basal levels of NRF2 and its downstream genes were upregulated upon addition of exogenous antioxidant NAC which was in concordance with existing studies which have demonstrated that NAC supplementation enhanced NRF2 expression [31]. However, depletion of glutathione should have further increased the NRF2 downstream genes to compensate and maintain the redox homeostasis but it failed. This could be attributed to already existing high ROS levels and lower NQ01 activity and GSH levels. Taken together, these evidences suggest that altering the levels of intracellular oxidative stress can modify the cellular response to anti-cancer drugs, such that induction of higher ROS levels sensitizes cancer cells to drug induced cell death.

Studies have showed that there is a connection between increased levels of antioxidant defenses and chemoresistance in cancers [32,33]. To adapt to the exogenous stress induced by the anti-cancer agents, cancer cells upregulate the antioxidant genes which allows them to survive under harsh conditions. Even though FDA approved Cisplatin and Oxaliplatin has been considered as the most active drugs traditionally, not all the patients benefit from platinum based chemotherapy. All the above evidences convey that there is a difference in the sensitivity of cervical cancers to anti-cancer drugs. Cisplatin and Oxaliplatin induced different levels of ROS and showed variations in the antioxidant regulatory genes which can contribute to the differential sensitivity displayed by cervical cancers to treatment. Chemoresistance is undoubtedly a multifactorial process and a lot of factors involved in the cell survivability pathways are likely to contribute. A better elucidation of these pathways and how they affect the differential responses to anti-cancer drug treatment can give the scientific audience an understanding of the molecular events leading to chemoresistance and may further help in targeted therapy of patients enduring from cervical cancers.

\section{Conclusion}


This study examined the differential responses of three cervical cancer cell lines and their link with the genes involved in the antioxidant regulators. The data clearly indicated that the variation in the levels of NRF2 and its downstream genes was responsible for the differences in the sensitivities of the cancer cells as well the induction of different ROS profiles. Of the three cell lines, C33A was the most resistant cell line for both cisplatin and oxaliplatin. C33A cells had high levels of NRF2 pathway genes, high NQ01 enzyme activity and high GSH levels when compared to HeLa and SiHa cells. Owing to the low NRF2 pathway genes, high amounts of ROS was induced in HeLa cells upon treatment with anti-cancer drugs. Finally, modifying the internal levels of oxidative stress demonstrated how it can bring about the change in the antioxidant regulatory genes which could in turn decide how the cell responds to anti-cancer treatment.

\section{Materials And Methods}

\section{Cell Culture and reagents}

The cells used in the present study: C33A, SiHa and HeLa representing the carcinoma of cervix were obtained from National Centre for Cell Science (NCCS), Pune, India. Cells were cultured in Dulbecco's Modified Eagle Medium (HiMedia, \#AL111) supplemented with 10\% South-American origin Fetal Bovine Serum (Gibco, Life technologies, \#26140-079), 2mM Glutamax (Gibco, Life technologies, \#35050-061) and $0.5 \mathrm{mg} / \mathrm{mL}$ Penicillin-Streptomycin (Gibco, Life technologies, \#1540-122) at 370C and 5\% CO2. All the cell lines were verified by HPV status using nested PCR [34] and were routinely checked for mycoplasma contamination by staining with Hoechst dye.

\section{Cell Treatment and Cytotoxicity Assay}

A total $1 \times 104$ cells per well were seeded in a 96-well plate for 24-32 hours. Once the cells reached a confluency of about $70 \%$, they were treated with various concentrations of Cisplatin and Oxaliplatin (3.12, $6.25,12.5,25,50,100$ and $200 \mu \mathrm{M})$ in triplicates. Control cells received just plain media in place of the drug. Following the treatment cells were incubated at $37^{\circ} \mathrm{C}$ with $5 \% \mathrm{CO} 2$ for 48 hours. The invitro cytotoxicity of the anti-cancer agents were determined using Sulforhodamine B (SRB) assay as per Skehan $\mathrm{P}$ et al, 1990 [35]. The drug treatment was repeated for three independent experiments. Cell viability was calculated as described in the literature [36].

\section{RNA extraction and cDNA synthesis}

A total of $0.5 \times 10^{4}$ cells per well were cultured in a 6-well plate and grown till confluent. NAC or BSO was added as indicated. Cell pellets were collected from the confluent wells after stipulated period of treatment and subjected to RNA extraction by TRIzol reagent (Ambion, Life technologies, \#AM9930). The extracted RNA was quantified using a Nano-drop spectrophotometer (DeNovix) and quality was assessed by electrophoresing on bleach agarose gel. Briefly, $1 \mu \mathrm{g}$ of total RNA from each sample was reverse transcribed using High-Capacity cDNA Reverse Transcription Kit (Applied Biosystems, \#4368814). 


\section{Quantitative real-time PCR}

To validate the differential expression of selected genes in the mRNA from all the three cell lines, q-RTPCR was performed with Rotor-Gene Q (Qiagen, Hilden, Germany) using the manufacturer's recommended protocol. Five microliters of cDNA $(10 \mathrm{ng} / \mu \mathrm{L})$ was amplified using DyNAmo ColorFlash SYBR green qPCR master mix (ThermoFischer Scientific, \# FNZ416L) to assess the expression of NRF2 and the selected antioxidant (NQ01, GCLC, GCLM, HO-1) genes regulated by NRF2. The gene expression of NRF2's negative regulator KEAP1 was also analyzed. The comparative threshold cycle (Ct) method was used for the calculation of amplification fold as described by Livak KJ et al, 2008 [37]. The observed gene levels were normalized using internal control GAPDH gene. The primers used in the study are described in Table 1.

\section{NQ01 activity}

Cells $\left(1 \times 10^{5}\right)$ were seeded in $100 \mathrm{~mm}$ dishes and were grown until confluent. The confluent cells were collected using a cell scraper. The cells were then lysed using special lysis buffer ( $5 \%$ NP40, 100mM EDTA, Protease inhibitor cocktail) at $37^{\circ} \mathrm{C}$ for 30 minutes and the lysates were stored at $-80^{\circ} \mathrm{C}$ freezer. NQ01 activity assay was performed as per Prochaska et al. 1988 [38].

\section{GSH levels}

Total Glutathione levels were measured using enzymatic recycling method as per modified Tietze's method [39]. The cell lysates were acquired as described above and was immediately processed since GSH levels tend to decrease over time.

\section{Measurement of Reactive oxygen species (ROS) levels}

To analyze the basal levels of ROS as well as the amount of ROS in each of cervical cancer cell line after the treatment with chemotherapeutic drugs, the cells were subjected to evaluation of intracellular ROS assay [40]. A total $1 \times 10^{4}$ cells per well were seeded in a 96-well plate and maintained inside an incubator at $37^{\circ} \mathrm{C}$ and $5 \% \mathrm{CO}_{2}$. Once the cells were $70 \%$ confluent, they were treated with cisplatin and oxaliplatin $(3.12,6.25,12.5,25,50,100$ and $200 \mu \mathrm{M})$ for 48 hours and subsequently with $250 \mu \mathrm{M}$ of H2O2 for 1 hour which served as an inducer of ROS (positive control). The cells were washed with 1x PBS and incubated with $10 \mu \mathrm{m}$ of 2', 7'-dichlorodihydrofluorescein diacetate (H2DCFDA) fluorescent probe (Sigma-Aldrich, \# D6883). The plates were incubated at $37^{\circ} \mathrm{C}$ inside a $\mathrm{CO}_{2}$ incubator for 30 minutes. The fluorescence was measured at $488 \mathrm{~nm} / 525 \mathrm{~nm}$ using a multimode plate reader (EnSpire, Perkin Elmer).

\section{Statistical analysis}

All assays were repeated three times and the data were tabulated for calculation Graph Pad Prism software (version 5.0). One way ANOVA followed by Tukey's post hoc test was performed to analyze the 
level of significance between the cell lines and treatment. A p value of $\leq 0.05$ was regarded as significant.

\section{Declarations}

\section{Funding}

No funding was received for conducting this study.

\section{Conflict of Interests}

The authors have no conflicts of interest to declare that are relevant to the content of this article.

\section{Availability of data}

The data that support the findings of this study are available on request from the corresponding author.

\section{Author contributions}

Conceptualization: Pushkal Sinduvadi Ramesh and Devananda Devegowda; Data curation, Formal analysis, Methodology: Pushkal Sinduvadi Ramesh, Sharanya Raja, Shwethambari Harave Udayakumar, and Shruthi Chandrashekar; Project administration, Resources and Supervision: Suma M Nataraj and Devananda Devegowda; Writing- original draft: Pushkal Sinduvadi Ramesh; Writing- review \& editing: Suma M Nataraj and Devananda Devegowda; Final approval of the manuscript: Pushkal Sinduvadi Ramesh, Sharanya Raja, Shwethambari Harave Udayakumar, Shruthi Chandrashekar, Suma M Nataraj and Devananda Devegowda

\section{Ethics approval}

Not applicable

\section{Consent to participate}

Not applicable

\section{Consent for publication}

Not applicable

\section{References}

1. Arbyn M, Weiderpass E, Bruni L, de Sanjosé S, Saraiya M, Ferlay J, Bray F. Estimates of incidence and mortality of cervical cancer in 2018: a worldwide analysis. The Lancet Global Health. 2020 Feb 1;8(2):e191-203. DOI: https://doi.org/10.1016/S2214-109X(19)30482-6 
2. de Martel C, Georges D, Bray F, Ferlay J, Clifford GM. Global burden of cancer attributable to infections in 2018: a worldwide incidence analysis. The Lancet Global Health. 2020 Feb 1;8(2):e18090. DOI: https://doi.org/10.1016/S2214-109X(19)30488-7

3. Almeida AM, Queiroz JA, Sousa F, Sousa Â. Cervical cancer and HPV infection: ongoing therapeutic research to counteract the action of E6 and E7 oncoproteins. Drug discovery today. 2019 Oct 1;24(10):2044-57. DOI: https://doi.org/10.1016/j.drudis.2019.07.011

4. Mellish KJ, Kelland LR, Harrap KR. In vitro platinum drug chemosensitivity of human cervical squamous cell carcinoma cell lines with intrinsic and acquired resistance to cisplatin. British journal of cancer. 1993 Aug;68(2):240-50. DOI: https://doi.org/10.1038/bjc.1993.322

5. Ebrahimi S, Soltani A, Hashemy SI. Oxidative stress in cervical cancer pathogenesis and resistance to therapy. Journal of cellular biochemistry. 2019 May;120(5):6868-77.

DOI: https://doi.org/10.1002/jcb.28007

6. Hong JH, Tsai CS, Lai CH, Chang TC, Wang CC, Chou HH, Lee SP, Hsueh S. Recurrent squamous cell carcinoma of cervix after definitive radiotherapy. International Journal of Radiation Oncology* Biology* Physics. 2004 Sep 1;60(1):249-57. DOI: https://doi.org/10.1016/j.jirobp.2004.02.044

7. Hasan S, Taha R, El Omri H. Current opinions on chemoresistance: An overview. Bioinformation. 2018;14(2):80. DOI: https://doi.org/10.6026/97320630014080

8. Fodale V, Pierobon M, Liotta L, Petricoin E. Mechanism of cell adaptation: when and how do cancer cells develop chemoresistance? Cancer journal (Sudbury, Mass.). 2011 Mar;17(2):89.

DOI: https://doi.org/10.1097/PPO.0b013e318212dd3d

9. Aoudjit F, Vuori K. Integrin signaling in cancer cell survival and chemoresistance. Chemotherapy research and practice. 2012;2012. DOI: https://doi.org/10.1155/2012/283181

10. Yang H, He L, Kruk P, Nicosia SV, Cheng JQ. Aurora-A induces cell survival and chemoresistance by activation of Akt through a p53-dependent manner in ovarian cancer cells. International journal of cancer. 2006 Nov 15;119(10):2304-12. DOI: https://doi.org/10.1002/ijc.22154

11. Mitsuishi Y, Motohashi H, Yamamoto M. The Keap1-Nrf2 system in cancers: stress response and anabolic metabolism. Frontiers in oncology. 2012 Dec 26;2:200.

DOI: https://doi.org/10.3389/fonc.2012.00200

12. Kobayashi M, Yamamoto M. Molecular mechanisms activating the Nrf2-Keap1 pathway of antioxidant gene regulation. Antioxidants \& redox signaling. 2005 Mar 1;7(3-4):385-94.

DOI: https://doi.org/10.1089/ars.2005.7.385

13. Jaramillo MC, Zhang DD. The emerging role of the Nrf2-Keap1 signaling pathway in cancer. Genes \& development. 2013 Oct 15;27(20):2179-91. DOI: https://doi.org/10.1101/gad.225680.113

14. Niture SK, Kaspar JW, Shen J, Jaiswal AK. Nrf2 signaling and cell survival. Toxicology and applied pharmacology. 2010 Apr 1;244(1):37-42. DOI: https://doi.org/10.1016/j.taap.2009.06.009

15. Lau A, Villeneuve NF, Sun Z, Wong PK, Zhang DD. Dual roles of Nrf2 in cancer. Pharmacological research. 2008 Nov 1;58(5-6):262-70. DOl: https://doi.org/10.1016/j.phrs.2008.09.003 
16. de la Vega MR, Chapman E, Zhang DD. NRF2 and the hallmarks of cancer. Cancer cell. 2018 Jul 9;34(1):21-43. DOI: https://doi.org/10.1016/j.ccell.2018.03.022

17. Singh A, Bodas M, Wakabayashi N, Bunz F, Biswal S. Gain of Nrf2 function in non-small-cell lung cancer cells confers radioresistance. Antioxidants \& redox signaling. 2010 Dec 1;13(11):1627-37. DOI: https://doi.org/10.1089/ars.2010.3219

18. Singh A, Boldin-Adamsky S, Thimmulappa RK, Rath SK, Ashush H, Coulter J, Blackford A, Goodman SN, Bunz F, Watson WH, Gabrielson E. RNAi-mediated silencing of nuclear factor erythroid-2-related factor 2 gene expression in non-small cell lung cancer inhibits tumor growth and increases efficacy of chemotherapy. Cancer research. 2008 Oct 1;68(19):7975-84. DOI: https://doi.org/10.1158/00085472.can-08-1401

19. Zhang M, Zhang C, Zhang L, Yang Q, Zhou S, Wen Q, Wang J. Nrf2 is a potential prognostic marker and promotes proliferation and invasion in human hepatocellular carcinoma. BMC cancer. 2015 Dec;15(1):1-2. DOI: https://doi.org/10.1186/s12885-015-1541-1

20. Tong YH, Zhang B, Yan YY, Fan Y, Yu JW, Kong SS, Zhang D, Fang L, Su D, Lin NM. Dual-negative expression of Nrf2 and NQ01 predicts superior outcomes in patients with non-small cell lung cancer. Oncotarget. 2017 Jul 11;8(28):45750. DOI: https://doi.org/10.18632/oncotarget.17403

21. Harvey CJ, Thimmulappa RK, Singh A, Blake DJ, Ling G, Wakabayashi N, Fujii J, Myers A, Biswal S. Nrf2-regulated glutathione recycling independent of biosynthesis is critical for cell survival during oxidative stress. Free Radical Biology and Medicine. 2009 Feb 15;46(4):443-53.

DOI: https://doi.org/10.1016/j.freeradbiomed.2008.10.040

22. Oh ET, Park HJ. Implications of NQ01 in cancer therapy. BMB reports. 2015 Nov;48(11):609. DOI: https://doi.org/10.5483/BMBRep.2015.48.11.190

23. Filippova M, Filippov V, Williams VM, Zhang K, Kokoza A, Bashkirova S, Duerksen-Hughes P. Cellular levels of oxidative stress affect the response of cervical cancer cells to chemotherapeutic agents. BioMed research international. 2014 Oct;2014. DOI: https://doi.org/10.1155/2014/574659

24. Kontostathi G, Zoidakis J, Makridakis M, Lygirou V, Mermelekas G, Papadopoulos T, Vougas K, Vlamis-Gardikas A, Drakakis P, Loutradis D, Vlahou A. Cervical cancer cell line secretome highlights the roles of transforming growth factor-beta-induced protein ig-h3, peroxiredoxin-2, and NRF2 on cervical carcinogenesis. BioMed research international. 2017 Feb 2;2017.

DOI: https://doi.org/10.1155/2017/4180703

25. Cho JM, Manandhar S, Lee HR, Park HM, Kwak MK. Role of the Nrf2-antioxidant system in cytotoxicity mediated by anticancer cisplatin: implication to cancer cell resistance. Cancer letters. 2008 Feb 18;260(1-2):96-108. DOI: https://doi.org/10.1016/j.canlet.2007.10.022

26. Ma X, Zhang J, Liu S, Huang Y, Chen B, Wang D. Nrf2 knockdown by shRNA inhibits tumor growth and increases efficacy of chemotherapy in cervical cancer. Cancer chemotherapy and pharmacology. 2012 Feb;69(2):485-94. DOI: https://doi.org/10.1007/s00280-011-1722-9

27. Itoh K, Mimura J, Yamamoto M. Discovery of the negative regulator of Nrf2, Keap1: a historical overview. Antioxidants \& redox signaling. 2010 Dec 1;13(11):1665-78. 
DOI: https://doi.org/10.1089/ars.2010.3222

28. Zhu H, Luo H, Zhang W, Shen Z, Hu X, Zhu X. Molecular mechanisms of cisplatin resistance in cervical cancer. Drug design, development and therapy. 2016;10:1885.

DOI: https://doi.org/10.2147/DDDT.S106412

29. Kim J, Keum YS. NRF2, a key regulator of antioxidants with two faces towards cancer. Oxidative Medicine and Cellular Longevity. 2016 Jan 1;2016. DOI: https://doi.org/10.1155/2016/2746457

30. Suzuki T, Yamamoto M. Molecular basis of the Keap1-Nrf2 system. Free Radical Biology and Medicine. 2015 Nov 1;88:93-100. DOI: https://doi.org/10.1016/j.freeradbiomed.2015.06.006

31. Jannatifar R, Parivar K, Roodbari NH, Nasr-Esfahani MH. The effect of N-acetyl-cysteine on NRF2 antioxidant gene expression in asthenoteratozoospermia men: a clinical trial study. International Journal of Fertility \& Sterility. 2020 Oct;14(3):171. DOI: https://doi.org/10.22074/ijfs.2020.44411

32. Jacob Victorino V, Pizzatti L, Michelletti P, Panis C. Oxidative stress, redox signaling and cancer chemoresistance: putting together the pieces of the puzzle. Current medicinal chemistry. 2014 Sep 1;21(28):3211-26. DOI: https://doi.org/10.2174/0929867321666140601164647

33. Traverso N, Ricciarelli R, Nitti M, Marengo B, Furfaro AL, Pronzato MA, Marinari UM, Domenicotti C. Role of glutathione in cancer progression and chemoresistance. Oxidative medicine and cellular longevity. 2013 Oct;2013. DOI: https://doi.org/10.1155/2013/972913

34. Ramesh PS, Devegowda DE, Naik PR, Doddamani PA, Nataraj SM. Evaluating the feasibility of nested PCR as a screening tool to detect HPV infection in saliva of oral squamous cell carcinoma subjects. J. Clin. Diagnostic Res. 2018 Jul 1;12.

DOI: https://doi.org/10.7860/JCDR/2020/44371.13825

35. Skehan P, Storeng R, Scudiero D, Monks A, McMahon J, Vistica D, Warren JT, Bokesch H, Kenney S, Boyd MR. New colorimetric cytotoxicity assay for anticancer-drug screening. JNCl: Journal of the National Cancer Institute. 1990 Jul 4;82(13):1107-12. DOI: https://doi.org/10.1093/jnci/82.13.1107

36. Anantharaju PG, Reddy DB, Padukudru MA, Chitturi CM, Vimalambike MG, Madhunapantula SV. Induction of colon and cervical cancer cell death by cinnamic acid derivatives is mediated through the inhibition of Histone Deacetylases (HDAC). PLoS One. 2017 Nov 30;12(11):e0186208.

DOI: https://doi.org/10.1371/journal.pone.0186208

37. Schmittgen TD, Livak KJ. Analyzing real-time PCR data by the comparative CT method. Nature protocols. 2008 Jun;3(6):1101-8. DOI: https://doi.org/10.1038/nprot.2008.73

38. Prochaska HJ, Santamaria AB. Direct measurement of NAD $(P) H$ : quinone reductase from cells cultured in microtiter wells: a screening assay for anticarcinogenic enzyme inducers. Analytical biochemistry. 1988 Mar 1;169(2):328-36. DOI: https://doi.org/10.1016/0003-2697(88)90292-8

39. Rahman I, Kode A, Biswas SK. Assay for quantitative determination of glutathione and glutathione disulfide levels using enzymatic recycling method. Nature protocols. 2006 Dec;1(6):3159-65. DOI: https://doi.org/10.1038/nprot.2006.378

40. Shailasree S, Venkataramana M, Niranjana SR, Prakash HS. Cytotoxic effect of p-coumaric acid on neuroblastoma, N2a cell via generation of reactive oxygen species leading to dysfunction of 
mitochondria inducing apoptosis and autophagy. Molecular neurobiology. 2015 Feb;51(1):119-30. DOI: https://doi.org/10.1007/s12035-014-8700-2

\section{Table}

Table 1: Primer sequences used for quantitative real time PCR in the study

\begin{tabular}{|lll|}
\hline GENE & SEQUENCE $\mathbf{5}^{\prime}$ to $\mathbf{3}^{\prime}$ & \\
\cline { 2 - 3 } & Forward & Reverse \\
NQ01 & TGCAGCGGCTTTGAAGAAGAAAGG & TCGGCAGGATACTGAAAGTTCGCA \\
GCLC & TTCAGCAGCATCCTCTCCACAG & GCATGCTGTTGCTGATACTGG \\
GCLM & ACTGACTTAGGAGCATAACTTACC & ACAGCACTTAGTAGGTTAACAGCACC \\
HMOX-1 & ATGGCCTCCCGTACCTCATC & TGTTGCGCTCAATCTCCTCCT \\
\hline KEAP-1 & CCATGAACGGTGGTGTGTT & GACCCTCTCGAGACGCAAC \\
\hline GAPDH & CGACCACTTTGTCAAGCTCA & AGGGGAGATTCAGTGTGGTG \\
\hline
\end{tabular}

\section{Figures}




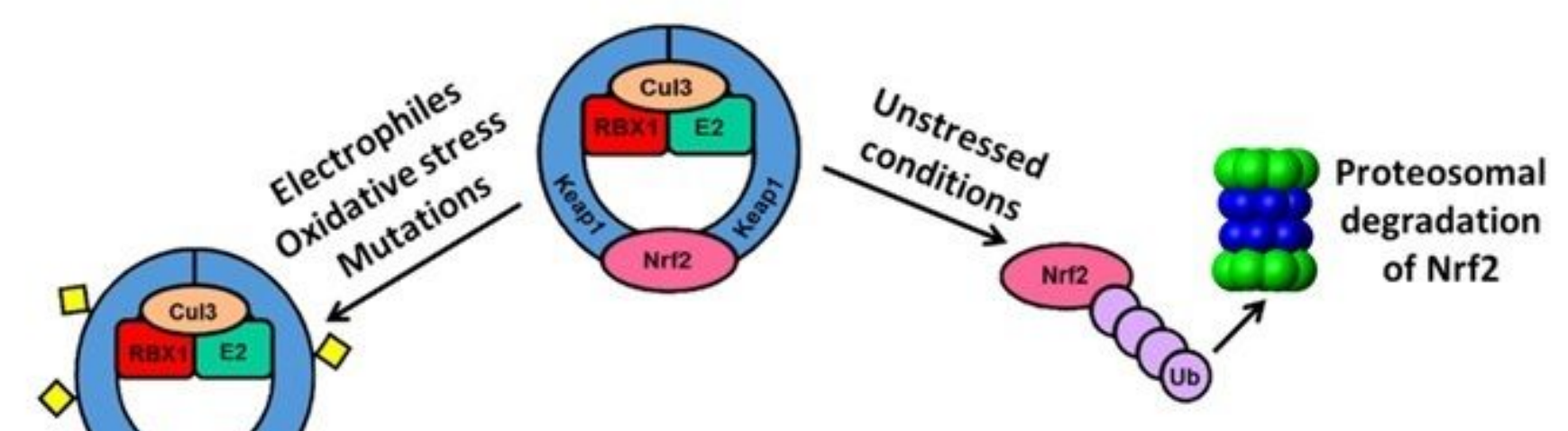

\section{KEAP1-NRF2 Pathway 1. GSH synthesis (GCLC, GCLM)}

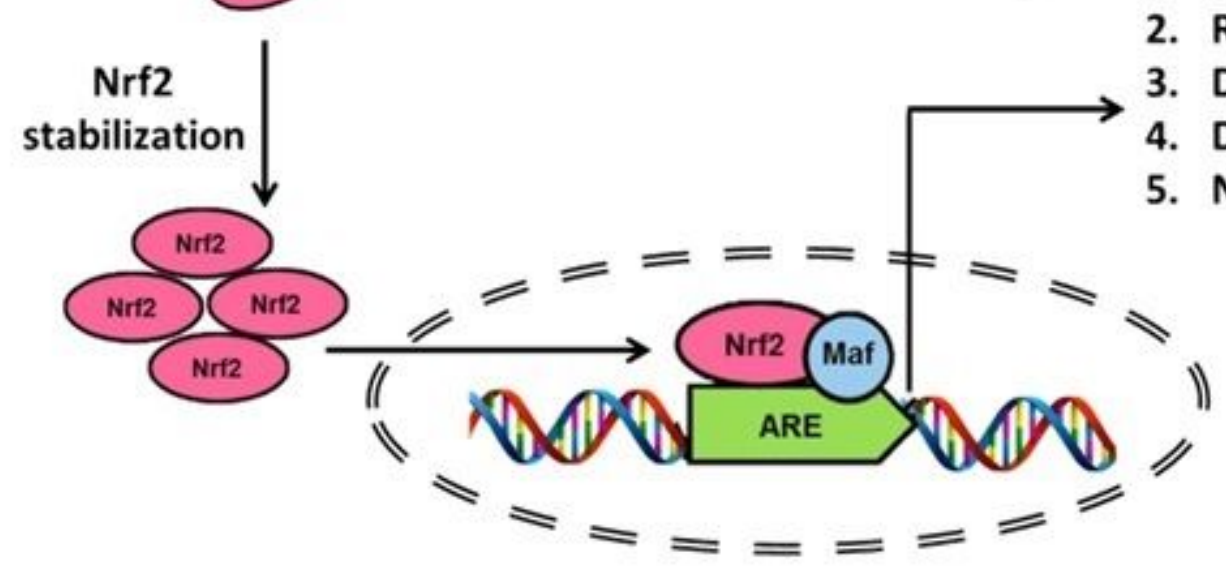

Figure 1

KEAP1/NRF2 pathway. It is a vital protective as well as cell survival pathway in response to oxidative and electrophilic stress. Under unstressed conditions, KEAP1 forms a complex with CUL3 and tightly regulates the NRF2 activity by ubiquitination and subsequent proteasomal degradation. On the other hand, upon exposure to oxidative stress, electrophiles and certain mutations, KEAP1 gets inactivated and NRF2 gets stabilized accumulating in the cytoplasm. This stabilized NRF2 translocates to nucleus where it forms a heterodimer with small Maf protein and binds to ARE leading to the induction of many cytoprotective genes 
2A

Dose response of C33A after $48 \mathrm{hrs}$ of treatment

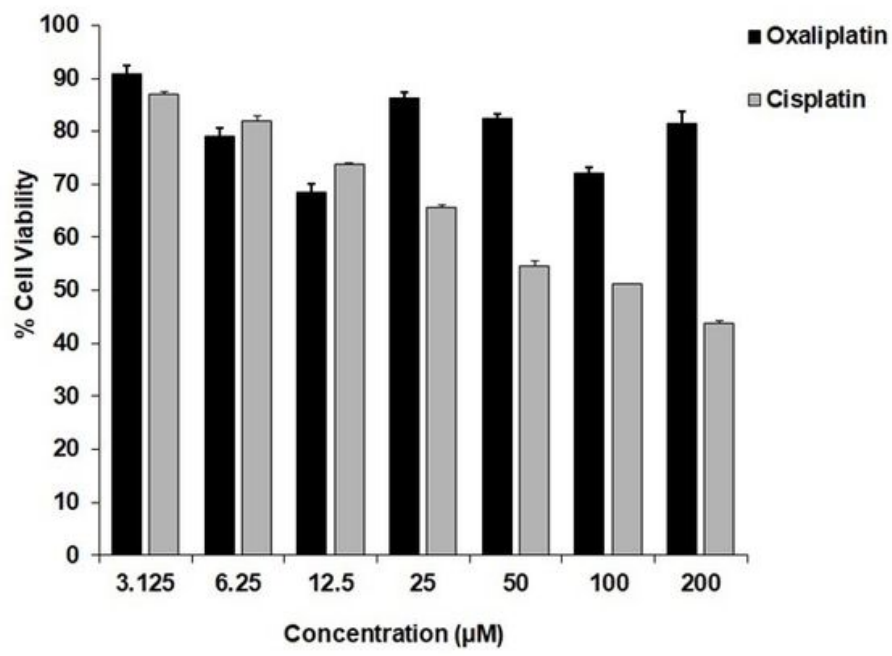

2C

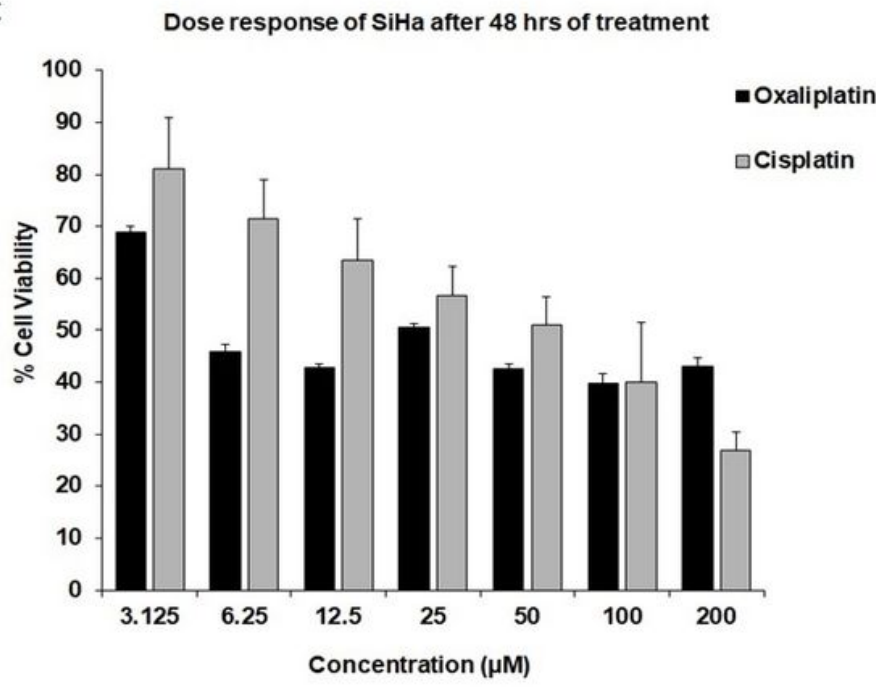

2B

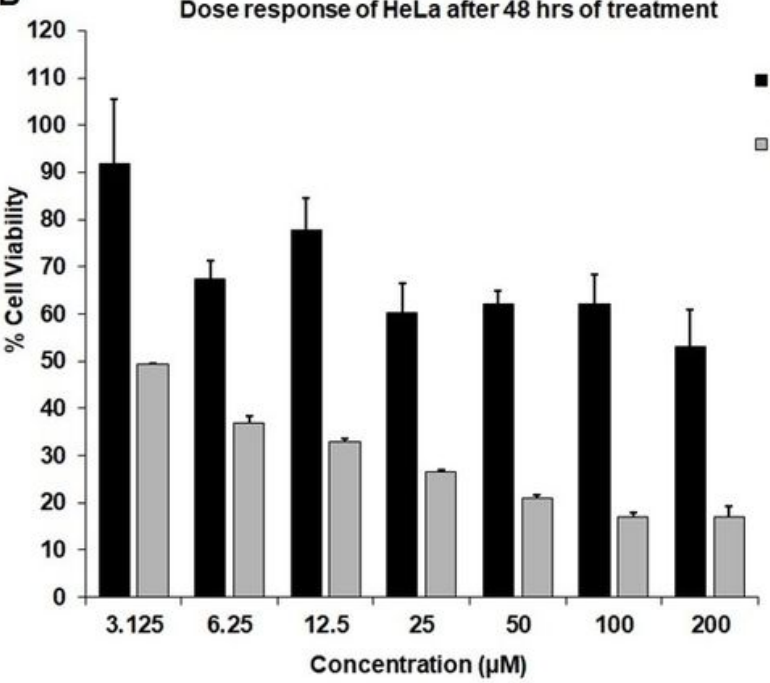

- Oxaliplatin

Cisplatin

\section{Figure 2}

Cell viability analysis using Sulforhodamine B (SRB) assay. Cell lines representing carcinoma of cervix: HeLa, SiHa and C33A were treated with different doses of Cisplatin and Oxaliplatin for 48 hours. Post treatment the cell viability analysis was done using SRB assay. (A) C33A cells showed resistance to oxaliplatin with more than $60 \%$ viable cells in all the doses. Cisplatin was comparatively more effective on C33A and the cells showed dose dependent response. (B) HeLa cells demonstrated a dose dependent cell death with cisplatin treatment and more than $70 \%$ of cells were killed at $25 \mu \mathrm{M}$. However, oxaliplatin treatment did not have any dose dependent effect on the cell viability. (C) SiHa cells even though showed dose dependent cell death with cisplatin however demonstrated some resistance to oxaliplatin. Each measurement was done in triplicate and error bars indicate the standard deviations of the means. 
3A

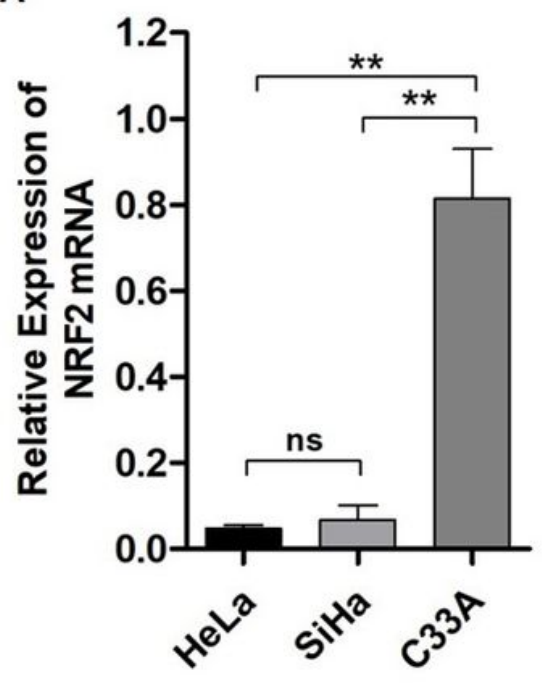

3C

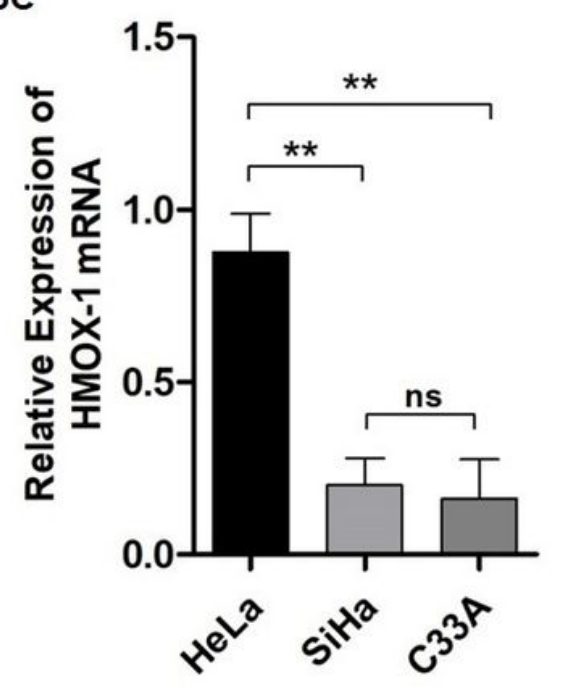

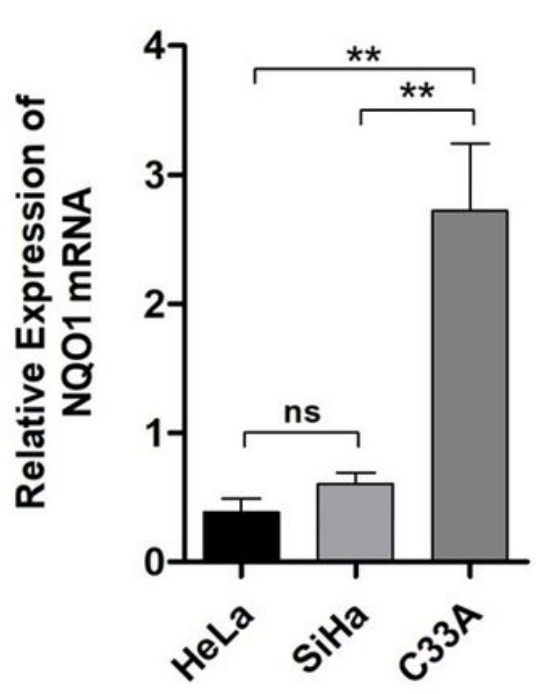

3D

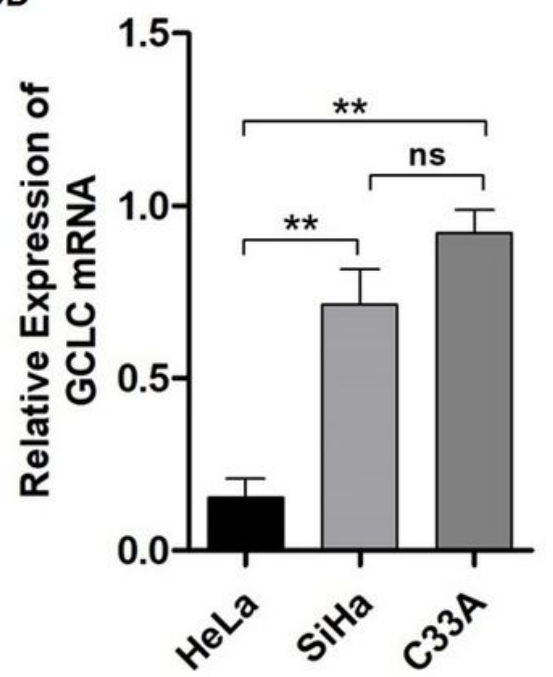

3B

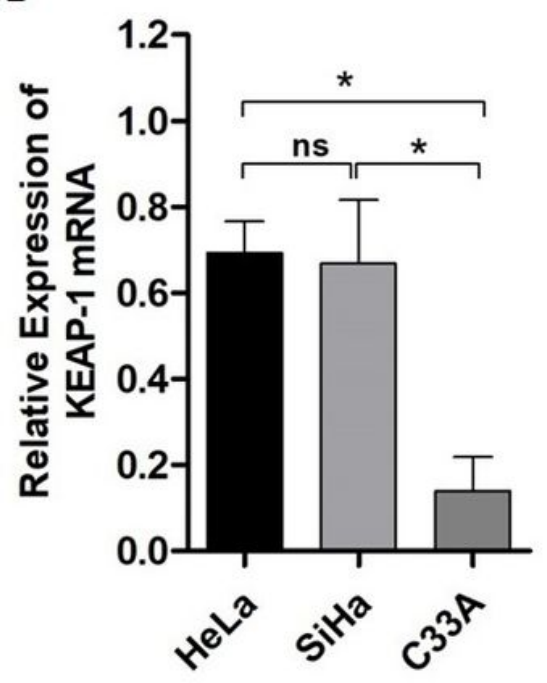

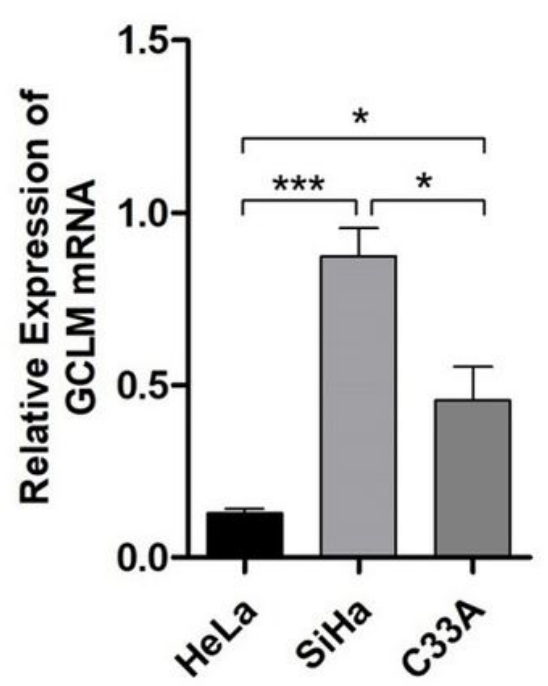

Figure 3

Quantification of mRNA levels of antioxidant regulatory genes regulated by NRF2. (A) C33A cells showed higher NRF2 and NQ01 gene levels compared to HeLa and SiHa cells. (B) HeLa and SiHa cells did not show any significant difference in the KEAP-1 gene levels however, C33A had significant downregulated levels of KEAP-1 when compared to both HeLa and SiHa. (C) HMOX-1 gene levels were significantly higher in HeLa cells when compared to both SiHa and C33A cells. (D) GCLC and GCLC gene levels were relatively higher in SiHa and C33A when compared to HeLa. The observed gene levels were normalized using internal control GAPDH gene. The experiments were performed three times and error bars indicate the standard deviations of the means. ${ }^{*} \mathrm{P}<0.05,{ }^{*} \mathrm{P}<0.01,{ }^{*} * \mathrm{P}<0.001$, ns $=$ not significant as determined by One-way ANOVA with Tukey's multiple comparison test. 
4A

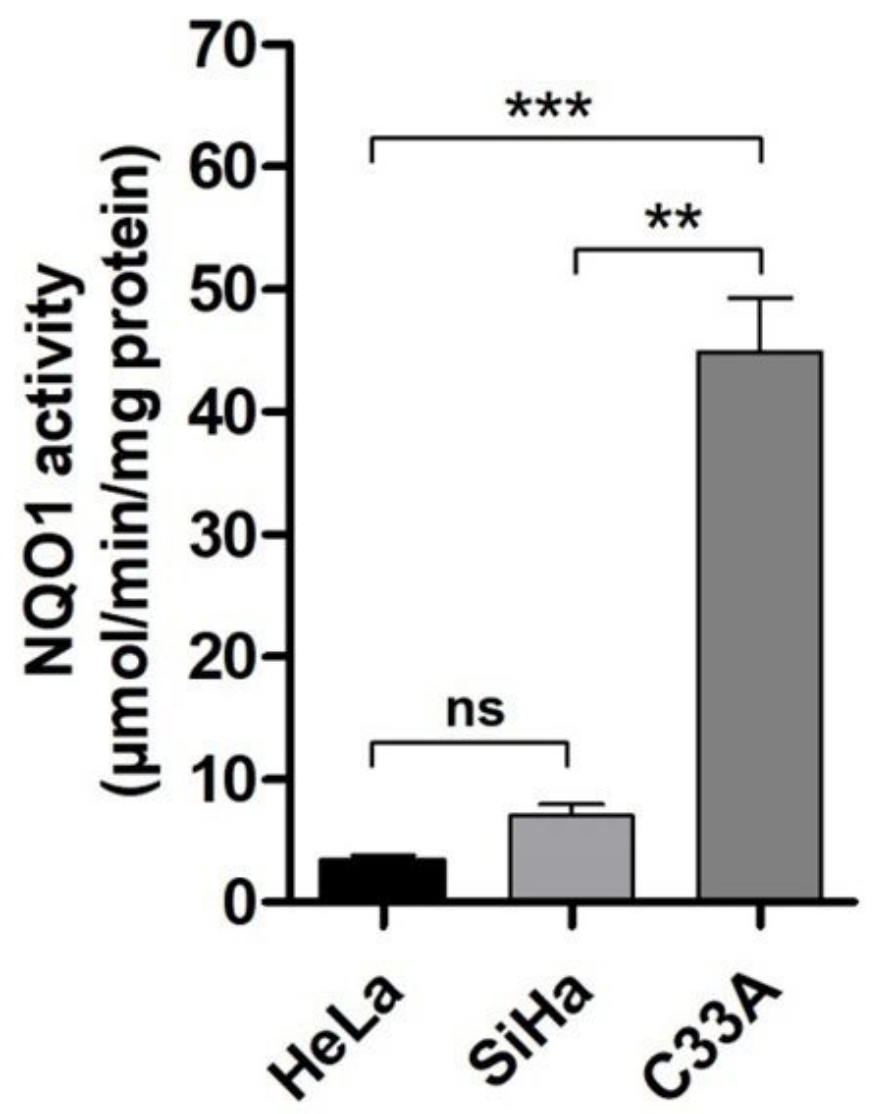

4B

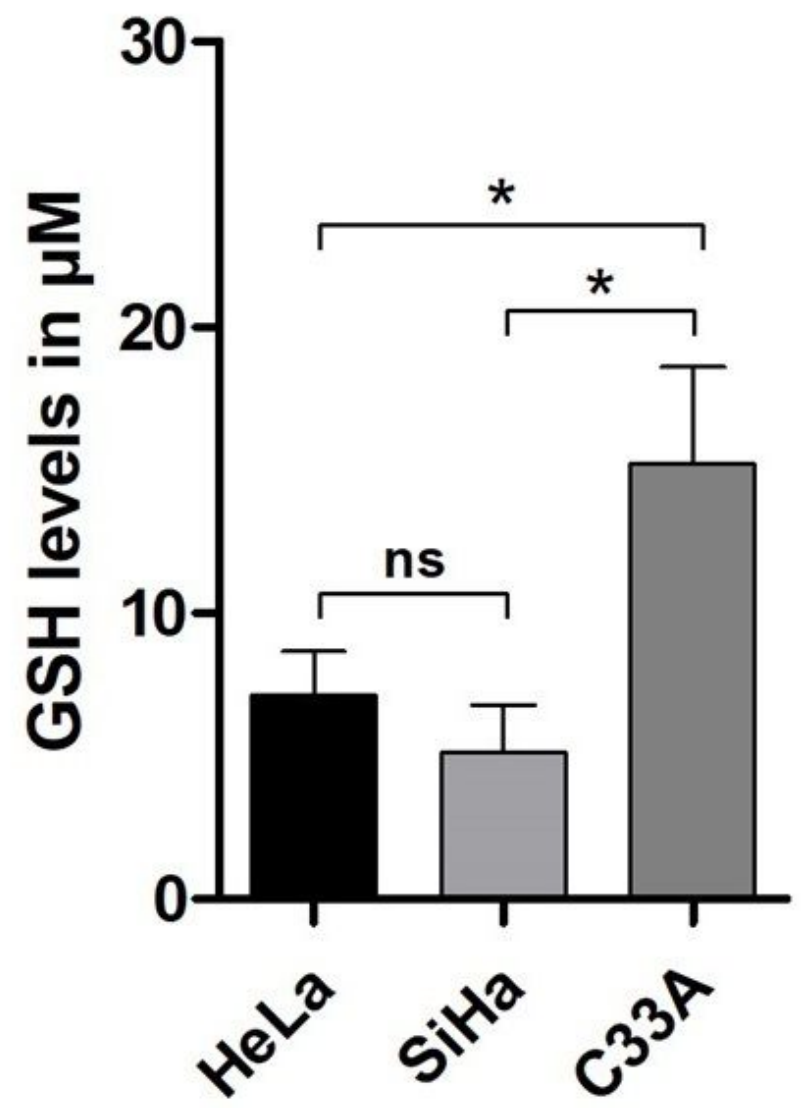

Figure 4

(A) NQ01 enzyme activity. A standard read-out parameter to assess NRF2 pathway is to measure NQ01 enzyme activity and we performed NQO1 activity in the cell lysates of SiHa, HeLa cells and C33A cells. C33A cells showed the highest NQ01 activity when compared to SiHa and HeLa cells. (B) Total GSH levels. C33A cells showed higher levels of total GSH compared to SiHa and HeLa cells. The experiments were performed three times and error bars indicate the standard deviations of the means. ${ }^{*} P<0.05$ ${ }^{*} \mathrm{P}<0.01,{ }^{* \star *} \mathrm{P}<0.001, \mathrm{~ns}=$ not significant as determined by One-way ANOVA with Tukey's multiple comparison test. 
$5 A$

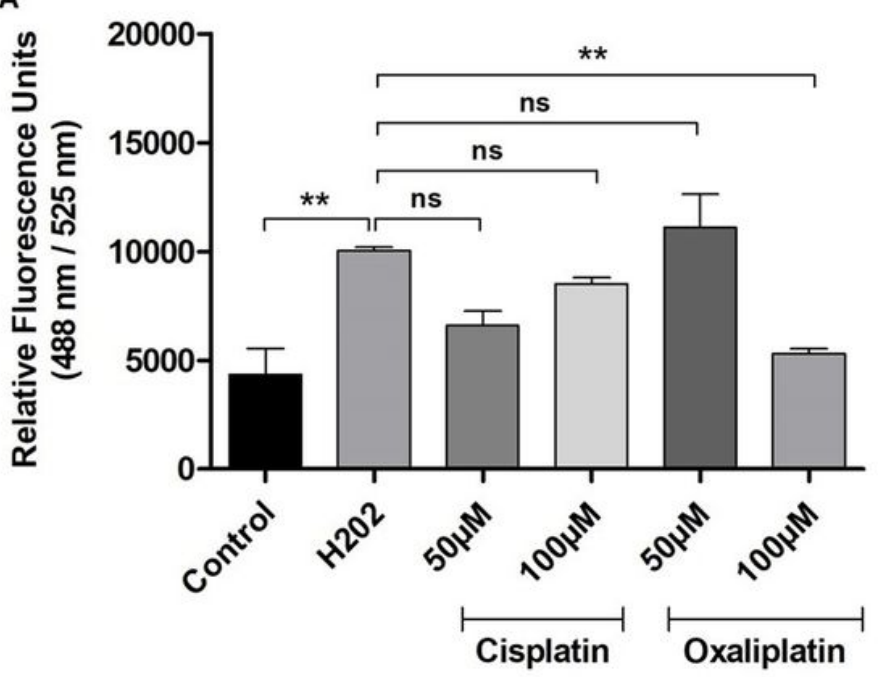

5B

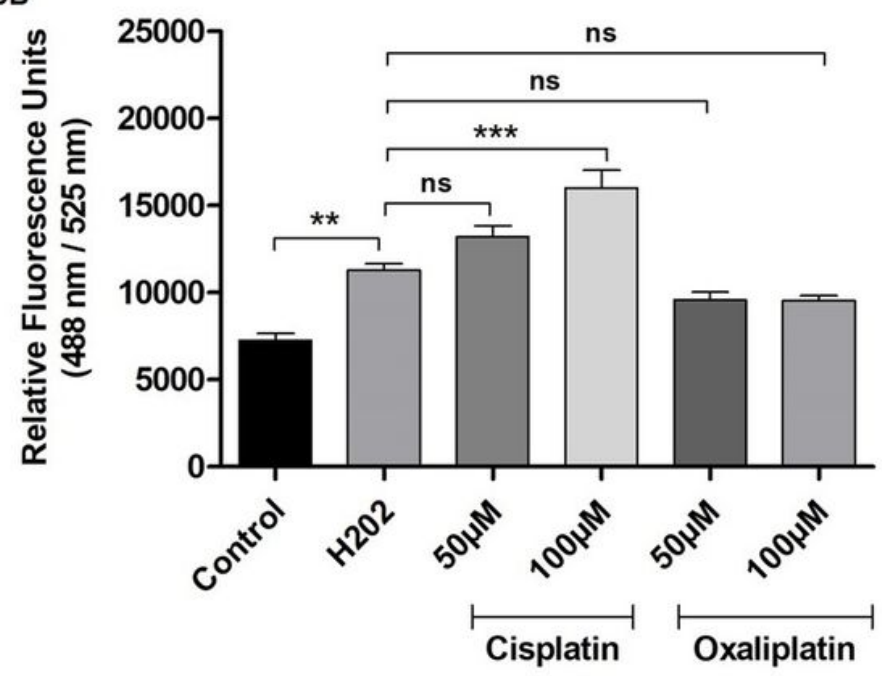

\section{Figure 5}

Levels of Reactive Oxygen Species (ROS). The differential expression of antioxidant related genes regulated by NRF2 is most likely to influence the baseline levels of Reactive Oxygen Species (ROS) in the cells. To test this idea, C33A and Hela were stained with H2DCFDA (2', 7- dicholofluorescin diacetate) after treating with selected drugs for 48 hrs and with $250 \mu \mathrm{M} \mathrm{H} 202$ as positive control subsequently for 1 hr. Cisplatin and Oxaliplatin were used in the concentrations of 50 and $100 \mu \mathrm{M}$. (A) ROS levels in C33A cells. (B) ROS levels in HeLa cells. The experiments were performed three times and error bars indicate the standard deviations of the means. ${ }^{* \star P} \mathrm{P}<0.01,{ }^{\star \star *} \mathrm{P}<0.001$, ns $=$ not significant. 

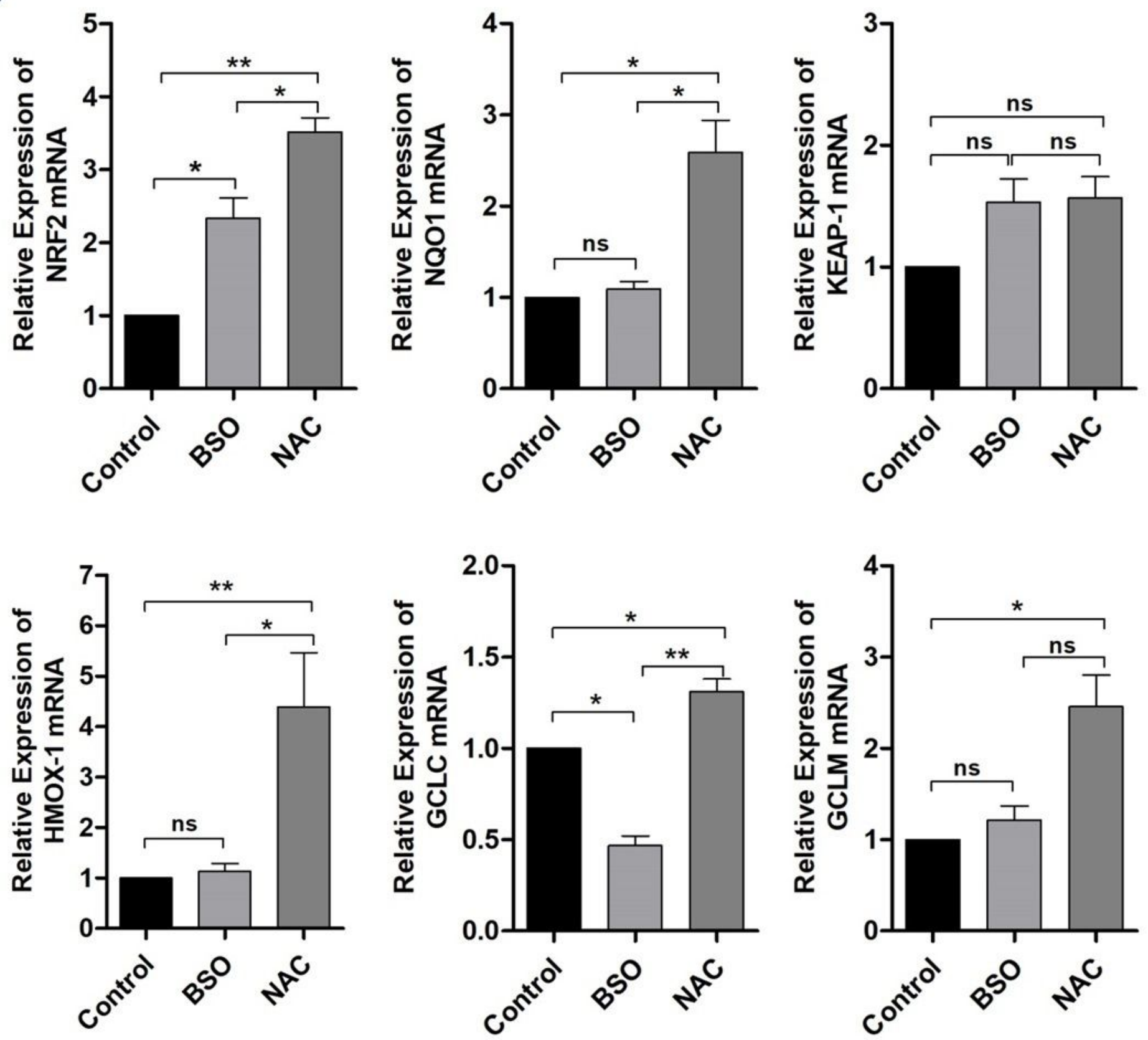

Figure 6

Quantification of mRNA levels of antioxidant regulatory genes regulated by NRF2 in HeLa cells after addition of $20 \mu \mathrm{M}$ of L-BSO (L-Buthionine Sulfoxamine, a glutathione inhibitor) and 4mM of NAC (N-Acetyl Cysteine, an exogenous antioxidant). The experiments were performed three times and error bars indicate the standard deviations of the means. ${ }^{*} P<0.05,{ }^{*} \mathrm{P}<0.01$, ns $=$ not significant 